\title{
The Optimization of Flush Atmospheric Data System Sensors Placement
}

\author{
Zhenxin Huang ${ }^{1, a}$, Zhenyu Jiang ${ }^{2, b}$ and Leyuan Sun ${ }^{2, c}$ \\ ${ }^{1}$ National University of Defense Technology, Changsha 410073, China \\ ${ }^{2}$ National University of Defense Technology, Changsha 410073, China \\ a18229793574@163.com, 'sly_nudt@126.com
}

Keywords: flush atmospheric data system, optimization of sensor placement, genetic algorithm.

\begin{abstract}
This paper is pointed at the optimization of flush atmospheric data system sensors placement, and developed the optimization methodology based on the non-dominated sorting genetic algorithm II (NSGA-II). First, the physical model of sensor system is analysed, then, designs the objective function and finds the best representation of the Pareto frontier, which makes you get the best sensor locations. Furthermore, simulations for different numbers of pressure points using these design methodology is performed based on CFD, the result showed that error of attack angle and dynamic pressure is less than $0.2 \%$, which identifies the availability of the methodology.
\end{abstract}

\section{Introduction}

With the development of aeronautics and astronautics and high-performance aircraft, precise air data are necessary to complete challenging high maneuvering tasks [1]. The work theories of FADS embody that the flight parameters are solved in terms of the pressures measured by the build-in sensors on the vehicle surfaces, and the flight parameters, such as angle of attack, angle of side-slip, Mach number and dynamic pressure are inferred from the aerodynamic model [2,3], The design concept of the flush air data sensing system (FADS) was presented by the American National Aeronautics and Space Administration in the 1960s in order to meet the control requirements of the space shuttle [4,5]. FADS has some significant advantages over traditional sensors [6,7]. However, in spite of observations that different port configurations can vastly affect the effectiveness of the estimation and the increasingly prevalent use, the methods for FADS design and sensor arrangement remain rudimentary. Design of the placement of the sensors on the vehicle fore-body have always been placed in symmetrical annular or cruciform patterns relied on engineering judgment and heuristic techniques rather than computationally based rationale [8]. For instance, FADS has been presented on Viking landers [9,10] and F-18 Hornet Attacker in cruciform patterns [11]. In this paper, according to the model between sensor measurements and estimate parameters, designs the objective function and finds the best representation of the Pareto frontier based on performance of estimate progress. Gets the best representation of the Pareto frontier form NSGA-II and makes a simulation to identify the availability of the optimization methodology.

\section{Algorithm Pressure Model}

The FADS Pressure Model (FPM) primarily combined Potential Flow Theory and Newton formula by the use of shaped pressure coefficient $\varepsilon$ to determine the air data state of a vehicle from differential pressures [12]. $\varepsilon$ is chosen by considering the factors of compress effect and aerodynamic configuration, it indicates the shaped pressure coefficient determined by the flight Mach and angle of attack and angle of side-slip $f\left(\alpha_{e}, \beta_{e}, M_{\infty}\right)$, the function can be known before flying. Then the algorithm pressure model of FADS can be written by [13]:

$$
p_{i}=p(\theta)=q_{c}\left(\cos ^{2} \theta_{i}+\varepsilon \sin ^{2} \theta_{i}\right)+p_{\infty}
$$

Where $p_{i}$ is the pressure of any pressure ports, $i=1,2 \cdots n, \theta_{i}$ is airflow angle of incidence, which can be known from[14]: 


$$
\cos \theta_{i}=\cos (\alpha) \cos (\beta) \cos \left(\lambda_{i}\right)+\sin (\beta) \sin \left(\phi_{i}\right) \sin \left(\lambda_{i}\right)+\sin (\alpha) \cos (\beta) \cos \left(\phi_{i}\right) \sin \left(\lambda_{i}\right)
$$

Where $\lambda_{i}$ indicates the cone angle, and $\phi_{i}$ denotes the circumferential angle as Figure 1 shows:

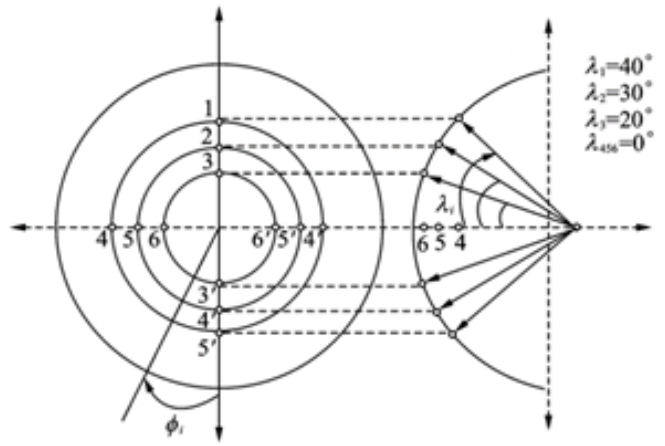

Fig. 1 Two or more references

Take the equation (2) to the equation (1), the function between sensor measurements and the estimate parameters can be written by:

$$
p_{i}=f\left(q_{c}, p_{\infty}, \alpha, \beta, \varepsilon, \lambda_{i}, \phi_{i}\right)
$$

The pressure $p_{i}$ will be decided by the position of pressure port $\left(\phi_{i}, \lambda_{i}\right)$, based on the interpolation or fitting to get the mapping relation between $p_{i}$ and $\left(\phi_{i}, \lambda_{i}\right)$, transform equation (3) to:

$$
g\left(\lambda_{i}, \phi_{i}\right)=f\left(q_{c}, p_{\infty}, \alpha, \beta, \varepsilon\right)
$$

Check the chart to confirm the value of shaped pressure coefficient $\varepsilon$ when given the initial condition and the flight Mach, take the vector of position $\left(\phi_{i}, \lambda_{i}\right)$ as input message, each estimate parameter can be expressed by:

$$
\begin{aligned}
& \alpha=f_{1}\left(\lambda_{1}, \phi_{1}, \lambda_{2}, \phi_{2} \cdots \lambda_{k}, \phi_{k}\right) \\
& \beta=f_{2}\left(\lambda_{1}, \phi_{1}, \lambda_{2}, \phi_{2} \cdots \lambda_{k}, \phi_{k}\right) \\
& q_{c}=f_{3}\left(\lambda_{1}, \phi_{1}, \lambda_{2}, \phi_{2} \cdots \lambda_{k}, \phi_{k}\right) \\
& p_{\infty}=f_{4}\left(\lambda_{1}, \phi_{1}, \lambda_{2}, \phi_{2} \cdots \lambda_{k}, \phi_{k}\right)
\end{aligned}
$$

\section{Optimization of Sensors Placement}

The optimization scheme of the sensor placement method is mainly based on NSGA-2 (Non-dominated Sorting Genetic Algorithm II) genetic algorithm [15]. The objective function is constructed by analyzing the physical model, and the satisfactory sensor configuration is obtained by finding in the objective function the optimal solution set that is closest to the Pareto boundary.

\subsection{Objective Function}

For flush air data sensing system, the sensors configuration optimization problem mentioned above is a multi-objective optimization process for multiple state variables need to be estimated in parallel during flight. Let the configuration has $\mathrm{V}$ pressure sensors, and their positions can be represented by $\left(\phi_{i}, \lambda_{i}\right), i=1,2 \cdots V$, then the objective function is designed as:

$$
\begin{aligned}
& \min \left(\sigma_{\alpha}, \sigma_{\beta}, \sigma_{q_{c}}, \sigma_{p_{\infty}}\right) \\
\sigma_{\alpha}= & g_{1}\left(\lambda_{1}, \phi_{1}, \lambda_{2}, \phi_{2} \cdots \lambda_{V}, \phi_{V}, \alpha_{0}\right) \\
\sigma_{\beta}= & g_{2}\left(\lambda_{1}, \phi_{1}, \lambda_{2}, \phi_{2} \cdots \lambda_{V}, \phi_{V}, \beta_{0}\right) \\
\sigma_{q_{c}}= & g_{3}\left(\lambda_{1}, \phi_{1}, \lambda_{2}, \phi_{2} \cdots \lambda_{V}, \phi_{V}, q_{c 0}\right) \\
\sigma_{p_{\infty}}= & g_{4}\left(\lambda_{1}, \phi_{1}, \lambda_{2}, \phi_{2} \cdots \lambda_{V}, \phi_{V}, p_{\infty 0}\right)
\end{aligned}
$$


where $\sigma_{\alpha}, \sigma_{\beta}, \sigma_{q_{c}}, \sigma_{p_{\infty}}$ are the differences between the estimated state parameters and the real state parameters, and $\alpha_{0}, \beta_{0}, q_{c 0}, p_{\infty 0}$ are the real state parameters.

Further more, for an aircraft with blunt body, there are the following constraints:

$$
0 \leq \lambda_{i} \leq \pi / 3 ; 0 \leq \phi_{i} \leq \pi
$$

\subsection{Optimization Algorithm}

NSGA-2 is based on the traditional genetic algorithm, and is often used to find the satisfactory solution of multi-objective optimization problem such as the FADS sensor configuration one mentioned above [16-18]. As is shown in Fig. 2, the algorithm consists of three basic steps, that is, initialization, classification and selection of inheritance. An initial sensor layout solution is generated randomly, and then those solutions are assigned to different tiers according to their objective function values. The diversity of the boundary is ensured by keeping basic crowding distance between various solutions. Offspring are generated based on the elite strategy of selecting the optimal parent, transition, mutation, etc. The iteration repeats until a preset termination condition is reached, and the last generation will be very close to the Pareto boundary $[19,20]$.

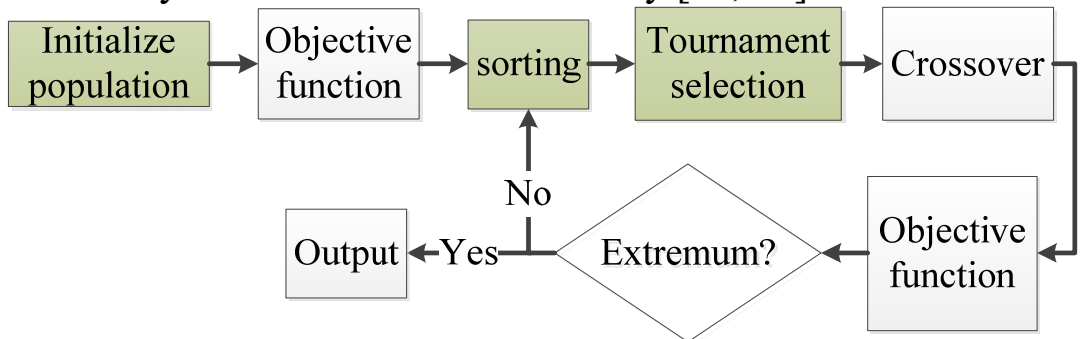

Fig. 2 Flow diagram of NSGA-II

\section{Simulation Analysis and Verifiction}

\subsection{Numerical Simulation}

As is shown in Fig.3, the flow field around a blunt-body aircraft is obtained by using a commercial software, and the simulated data will be used to verify the algorithm designed in Section 3.
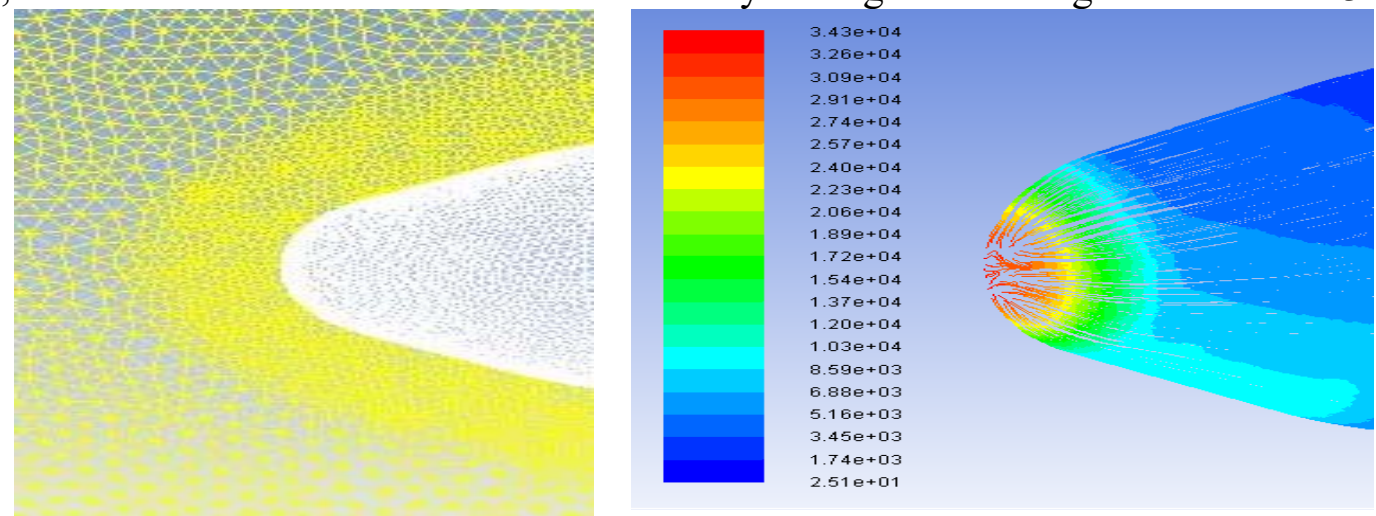

Fig. 3 Numerical simulation of blunt-body aircraft

The static pressure $P_{\infty}$ is referred to the international atmospheric data sheet, the total pressure and the dynamic pressure measured by the $i^{\text {th }}$ sensor located at $\left(\phi_{i}, \lambda_{i}\right)$ can be obtained by equation (9) using the total/static pressure relationship, where $\phi_{i}$ and $\lambda_{i}$ represent the cone angle and circumferential angle respectively.

$$
P_{t}=P_{\infty} \times\left(1+\frac{\gamma-1}{2} \times M a^{2}\right)^{\frac{\gamma}{\gamma-1}}
$$



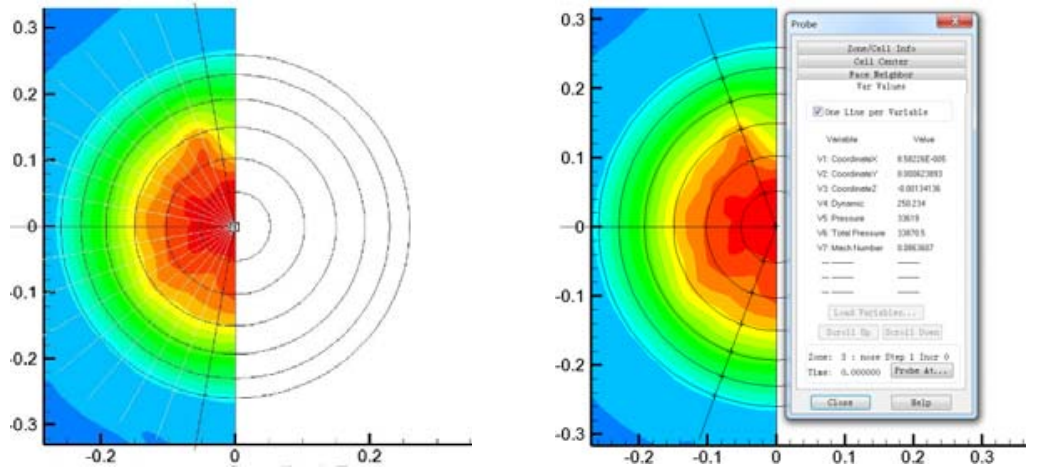

Fig. 4 Mesh generation of forebody by cone angle and circle angle

Considering the symmetry principle of the sensor layout, only those sensors located in the left half plane is optimized. To get the relationship between the sensor location and the pressure value, the head region is divided into the grid shown in Fig.4, where the flow field is divided into 6 regions (every 10 degrees) in cone angle direction and 18 regions (every 10 degrees) in circumference angel direction. The pressure value at each intersection is measured, and a surface chart describing the relationship is plotted as is shown in Fig.5, where axis $X$ represents the cone angle vector $\lambda_{i}$, axis $Y$ represents the circle angle vector $\phi_{i}$, and axis $Z$ represents the pressure value $p_{\left(\phi_{i}, \lambda_{i}\right)}$.

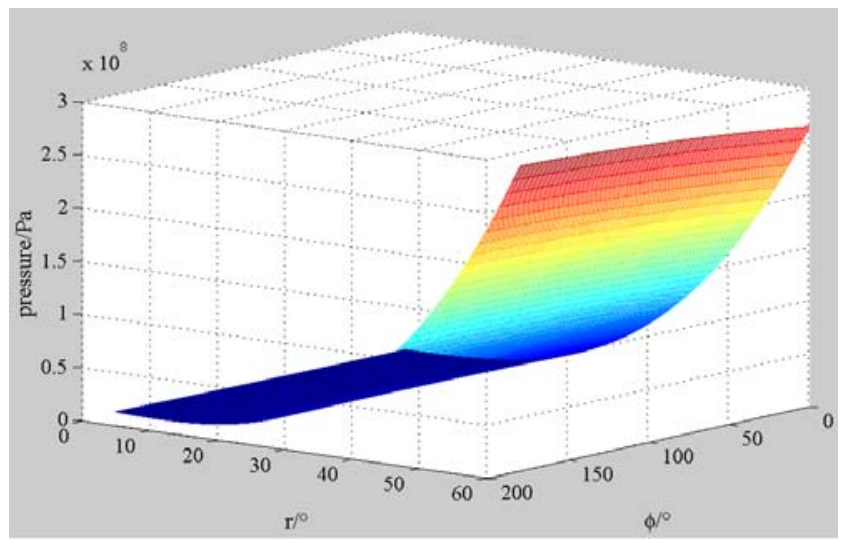

Fig. 5 Mapping relation between pressures and positions

The function $p_{i}=P\left(\phi_{i}, \lambda_{i}\right)$ describing the relationship between the pressure value $p_{i}$ and the position vector $\left(\phi_{i}, \lambda_{i}\right)$ can be fitted according to the statistics mentioned above. To simplify the calculation, only the angle of attack $\alpha$ and the dynamic pressure $q_{c}$ are validated and used to determine the objective functions $f\left(\sigma_{q_{c}}\right)$ and $f\left(\sigma_{\alpha}\right)$, and NSGA-2 is applied to find the satisfactory solution.

\subsection{Optimization Results}

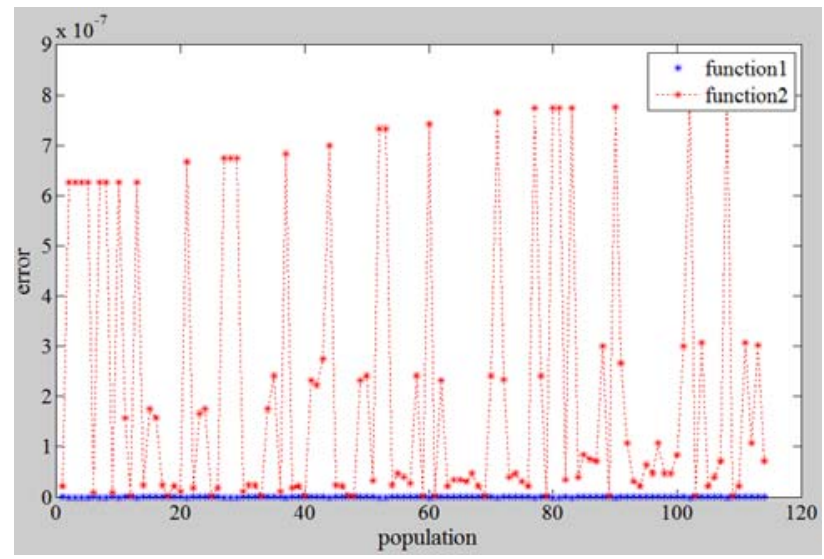

Fig. 6 Objective function value of six pressure ports 
Fig.6 shows trend of the objective functions of the 6-sensor layout optimization process, and the values are negligible with a maximum less than $9 \times 10^{-7}$. The second objective function can meet some mutations during optimization, but those solutions can be discarded.
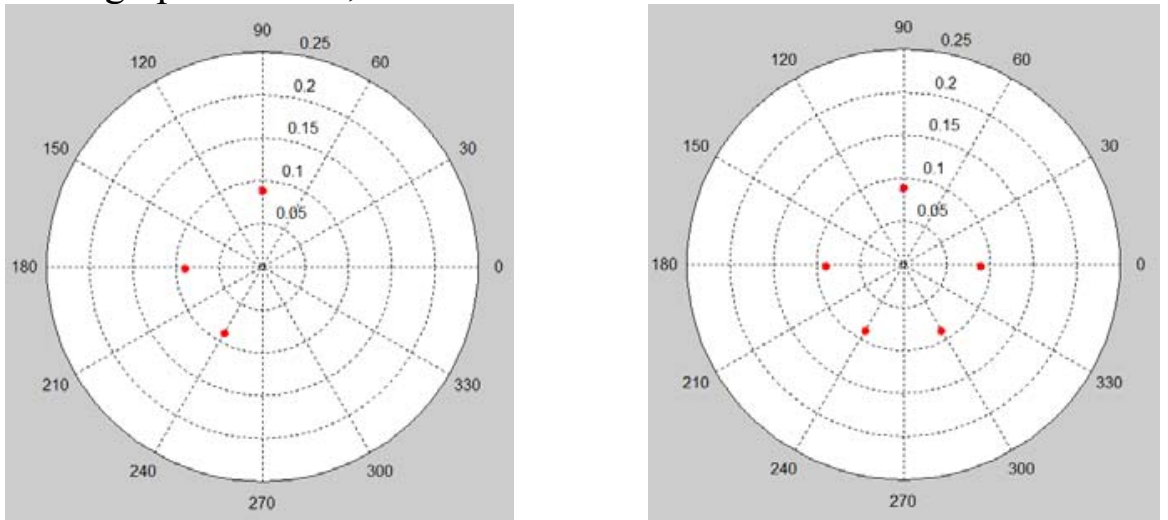

Fig. 7 Optimization result of six pressure ports

Having found the satisfactory solution in the left plane, the whole sensor configuration scheme can be determined according to the symmetry principle. Fig.7 shows the optimization results of the 6-sensor configuration, and the holes is distributed around a circle. Two coinciding points can be found on the central axis, and therefore can be replaced by a hole to realize further optimization.

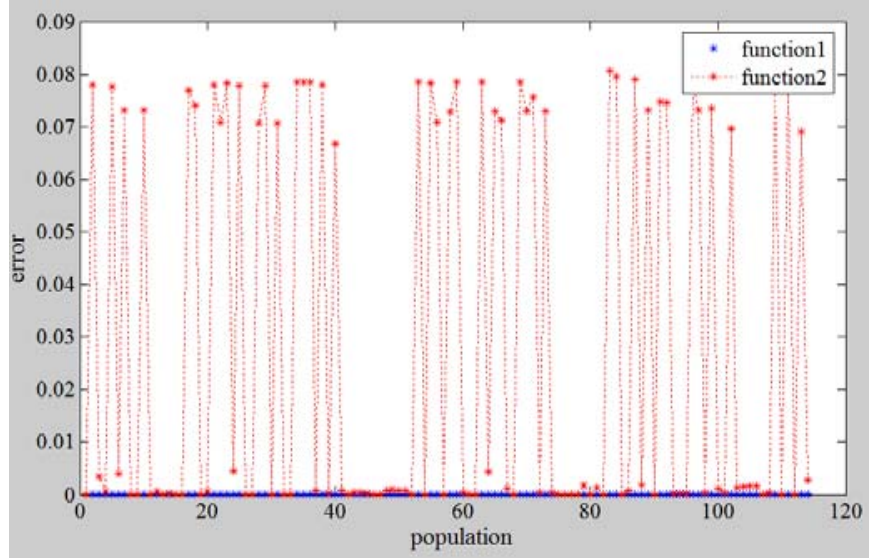

Fig. 8 Objective function value of eight pressure ports

The objective functions of the 8-sensor configuration method are shown in Fig.8, and compared with the previous 6-sensor configuration method, more oscillations of the second objective function appears, most of which are between 0 and 0.075 .
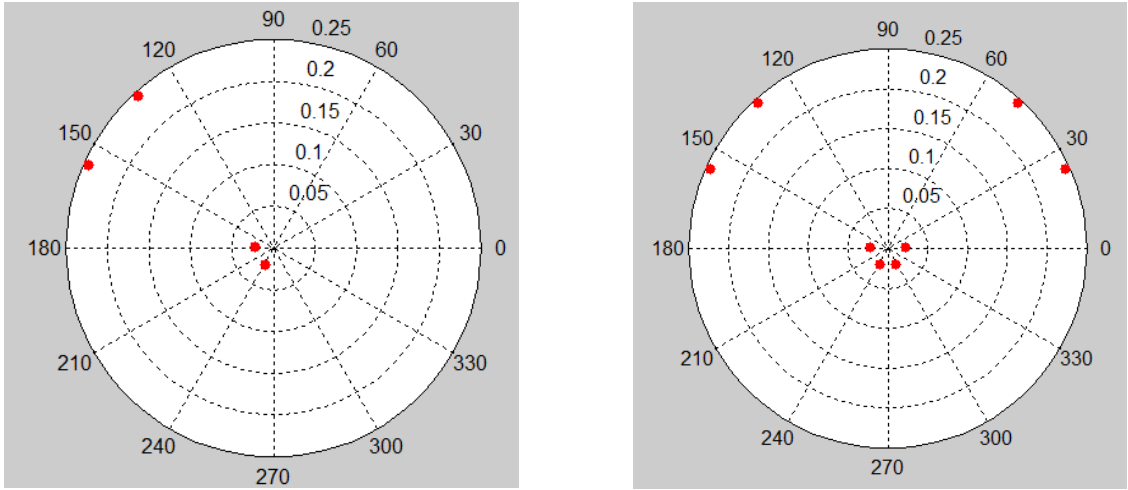

Fig. 9 Optimization result of eight pressure ports

Fig. 9 shows the optimization results of the 8 -sensor configuration. The holes is distributed around two circles of different sizes. Four points locate along the edge, and thus can be replaced by a hole to realize further optimization, reducing the sensor numbers to 5 . 


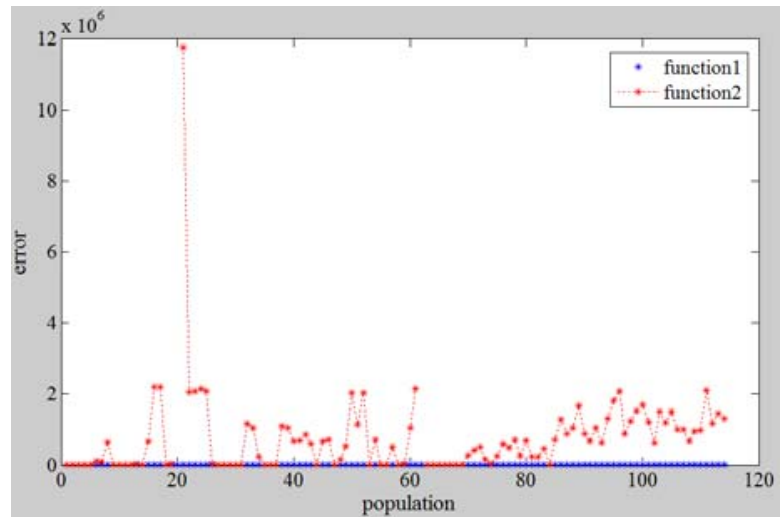

Fig. 10 Objective function value of ten pressure ports

The objective functions of the 10-sensor configuration method are shown in Fig.10, and the offset of the second objective function increased significantly. Therefore, we can draw a conclusion that larger numbers of the pressure sensors cannot guarantee better performance of the system.
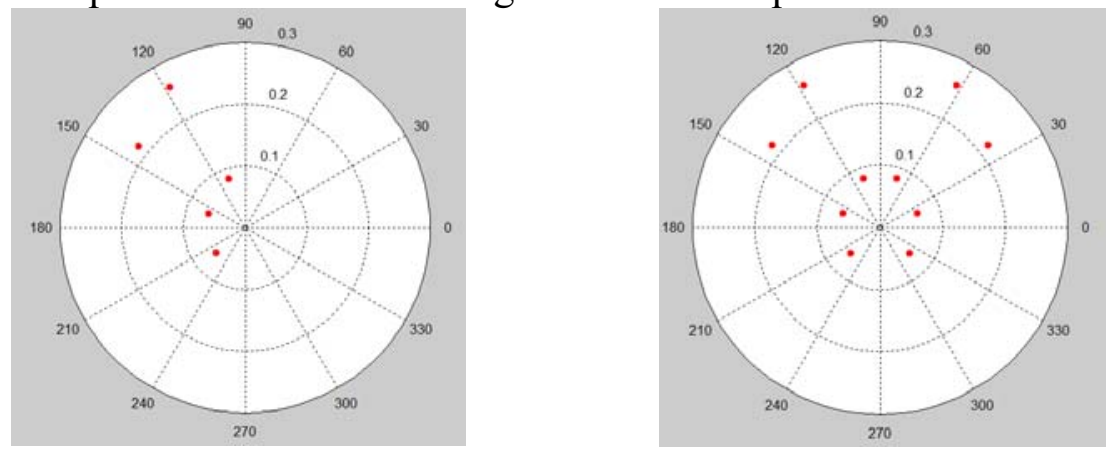

Fig. 11 Optimization result of ten pressure ports

Fig. 11 shows the optimization configuration of 10 sensors, and most pressure sensors are located around the apex of the head.

Table 1 shows the optimal solutions of different number of pressure sensors, and all estimation results are satisfactory. The estimation errors of the angle of attack are negligible, and the estimation errors of the dynamic pressure are less than $0.2 \%$. In practice, the 6-hole optimal layout and the 8-hole optimal layout can be further optimized into a 5-hole configuration, and by comparing the simulated results, the 8-hole solution is suggested.

Table 1 Estimate results of different sensor numbers

\begin{tabular}{|c|c|c|c|c|}
\hline & Parameter & Real Value & Estimate Value & Error \\
\hline \multirow{2}{*}{$\mathrm{V}=3$} & $\alpha$ & 0 & $0.74 \mathrm{E}-10$ & $7.4 \mathrm{E}-9 \%$ \\
\cline { 2 - 5 } & $q_{c}$ & 2269924 & 2272268 & $0.11 \%$ \\
\hline \multirow{2}{*}{$\mathrm{V}=4$} & $\alpha$ & 0 & $0.182 \mathrm{E}-13$ & $\begin{array}{c}1.8 \mathrm{E}-14 \\
\%\end{array}$ \\
\cline { 2 - 5 } & $q_{c}$ & 2269924 & 2271057 & $0.05 \%$ \\
\hline \multirow{2}{*}{$\mathrm{V}=5$} & $\alpha$ & 0 & $0.66 \mathrm{E}-8$ & $6.6 \mathrm{E}-9 \%$ \\
\cline { 2 - 5 } & $q_{c}$ & 2269924 & 2274323 & $0.19 \%$ \\
\hline
\end{tabular}

\section{Conclusions}

This paper is pointed at the optimization of sensor configuration, and analyzed the physical model of sensor system, designed the objective function and finds the best representation of the Pareto frontier based on NSGA-II. Furthermore, simulations for different numbers of pressure points using these design methodology is performed based on CFD, the result showed that error of attack angle and dynamic pressure is less than $0.2 \%$, which identifies the availability of the methodology. 


\section{References}

[1] Liu, Y.B.; Xiao, D.B.; Lu, Y.P. Comparative Study on a Solving Model and Algorithm for a Flush Air Data Sensing System. Sensors 2014, 14, 9210-9226.

[2] Larson, T.J.; Moes, T.R.; Siemers, P.M. Wind-Tunnel Investigation of a Flush Air data System at Mach Numbers from 0.7 to 1.4; NASA TM-101697; National Aeronautics and Space Administration, Washington, DC, America, 1990.

[3] Wang, P.; Xin, J. Application of the FADS System to Hypersonic Flight Vehicles with Sharp Wedged Fore-bodies. 18th AIAA/3AF International Space Planes and Hypersonic Systems and Technologies Conference 24 - 28 September 2012, Tours, France.

[4] Timothy, J.W. Shuttle entry air data system-an experimental investigation of calibration for ascent flight. In Proceedings of the 30th Aerospace Sciences Meeting \& Exhibit, Reno, NV, USA, 6-9 January 1992.

[5] Luo, Y.Z.; Zhang, J.; Tang, G.J. Survey of orbital dynamics and control of space rendezvous. Chin. J. Aeronaut. 2014, 27, 1-11.

[6] Li, H.N.; Pei, Q.Q.; Jiang, X.H. Sub-Spectrum sensing based on reputation in CRNS. Int. J. Innov. Comput. Inf. Control 2012, 8, 3705-3715.

[7] Ejaz, W.; Hasan, N.; Kim, H.S. SNR-based adaptive spectrum sensing for cognitive radio networks. Int. J. Innov. Comput. Inf. Control 2012, 8, 6095-6105.

[8] Soumyo Dutta; Robert D.B. Cramer-Rao Lower-Bound Optimization of Flush Atmospheric Data System Sensor Placement. JOURNAL OF SPACECRAFT AND ROCKETS Vol. 51, No. 6, November-December 2014.

[9] Ingoldby, R.; Michel, F.; Flaherty, T.; Doty, M.; Preston, B.; Villyard, K; and Steele, R.; Entry Data Analysis for Viking Landers 1 and 2, NASA CR-159388, 1976.

[10]Blanchard, R. C.; and Walberg, G. D. Determination of the Hypersonic-Continnum /Rarefied-Flow Drag Coefficient of the Viking Lander Capsule 1 Aeroshell from Flight Data, NASA TM-1793, 1980.

[11]Information on http://airforcescrapbook.blogspot.com/2008/02/usafscrapbook-update.html;

[12] Samy, I.; Postlethwaite, I.; Gu, D.W. Unmanned air vehicle air data estimation using a matrix of pressure sensors: A comparison of neural networks and look-up tables. Proc. Inst. Mech. Eng. Part G J. Aerosp. Eng. 2011, 225, 807-820.

[13]Rohloff, T.J.; Whitmore, S.A.; Catton, I. Fault-tolerant neural network algorithm for flush air data sensing. J. Aircraft 1999, 36, 541-549.

[14]Zhou, G.C.; Li, Q.D.; Guo, Y.M. A Highly Precise FADS (Flush Air-Data Sensing System) Algorithm. Journal of North western Polytechnical University Vol.32, No.3, June 2014.

[15]Deb, K. Multi-Objective Optimization Using Evolutionary Algorithms, Wiley, Hoboken, NJ, 2001, pp. 25-46.

[16]Dutta, S., Braun, R. D., and Karlgaard, C. D., Atmospheric Data System Sensor Placement Optimization for Mars Entry, Descent, and Landing, Journal of Spacecraft and Rockets, Vol. 51, No. 1, 2014, pp. 163-174.

[17]Deb, K., Pratap, A., Agarwal, S., and Meyarivan, T., A Fast and Elitist Multi-objective Genetic Algorithm: NSGA-II,'IEEE Transactions on Evolutionary Computation, Vol. 6, No. 2, 2002, pp. 182-197.

[18]Reyes-Sierra, M., and Coello, C. A., Multi-Objective Particle Swarm Optimizers: A Survey of the State-of-the-Art," International Journal of Computational Intelligence Research, Vol. 2, No. 3, 2006, pp. 287-308.

[19]Deb, K., and Agarwal, R., Simulated Binary Crossover for Continuous Search Space, Complex Systems, Vol. 9, April 1995, pp. 115-148.

[20]Beyer, H.-G., and Deb, K., On Self-Adaptive Features in Real Parameter Evolutionary Algorithms, IEEE Transactions in Evolutionary Computation, Vol. 5, No. 3, 2001, pp. 250-270. 